\title{
Composición química de aceites esenciales de hojas de Fridericia florida DC. y Fridericia chica (Bonpl.)
} Beatriz Devia Castilloa, Yudy Mahecha Jiméneza ${ }^{a}$ María Franco Nieves ${ }^{a}$, Javier
Matulevich Peláez ${ }^{a}$

\begin{abstract}
Resumen: Se identificó la composición química de los aceites esenciales de hojas frescas de Fridericia florida DC. L.G Lohman y Fridericia chica (Bonpl) (Bignoniaceae), obtenidos por destilación con arrastre de vapor. La identificación se realizó por cromatografía de gases acoplada a espectrometría de masas (CG-EM), comparación de índices de retención y espectros de masas reportados por la literatura. Se obtuvo un rendimiento de $0.033 \%$, y se identificó el $96.78 \%$ (Fridericia florida) y el $91.95 \%$ (Fridericia chica) de su composición total relativa. En Fridericia florida se identificaron 13 compuestos, de los cuales el mayoritario fue el limoneno (72.17\%). En Fridericia chica se identificaron 11 compuestos, de los cuales el mayoritario fue el germacreno D (63.77\%). Estos resultados contribuyen a la fitoquímica de ambas especies, al conocimiento de los componentes volátiles de especies nativas de uso tradicional y constituyen un aporte al género Fridericia, del que no se registran estudios sobre la composición de aceites esenciales.
\end{abstract}

Palabras Clave: Fridericia florida; Fridericia chica; CG-EM; monoterpenos; sesquiterpenos

Recibido: 3 de octubre de 2018

Evaluado: 21 de febrero de 2019

Aceptado: 7 de marzo de 2019

Disponible: 2 de marzo de 2020

Cómo citar: Devia Castillo, B. O., Mahecha Jiménez, Y., Franco Nieves, M. F., \& Matulevich Peláez, J. A. (2020). Composición química de aceites esenciales de hojas de Fridericia florida DC. y Fridericia chica (Bonpl.). Revista Facultad De Ciencias Básicas, 15(1), 63-70. https://doi. org/10.18359/rfcb.3676

a Facultad de Ciencias y Educación, Universidad Distrital Francisco José de Caldas, Carrera 4 \# 26 B-54, Bogotá, Colombia. 


\title{
Chemical Composition of the Essential Oil Leaves of Fridericia Florida DC. and Fridericia Chica (Bonpl.).
}

\begin{abstract}
This research project identifies the chemical composition of the essential oils extracted by steam distillation from fresh leaves of Fridericia florida DC L. G Lohman and Fridericia chica (Bonpl) (Bignoniaceae). The identification was performed by gas chromatography coupled to mass spectrometry (CG-EM). Then, the retention rates and mass spectra obtained were compared against the values reported by the literature. In this study, a yield of $0.033 \%$ was obtained, thus identifying $96.78 \%$ (Fridericia florida) and $91.95 \%$ (Fridericia chica) in its relative total composition. For Fridericia florida, 13 compounds were identified, with limonene being the major component (72.17\%). For Fridericia chica, 11 compounds were identified, with germacrene D being the major component (63.77\%). These results contribute to the phytochemistry of both species, identifying the volatile components in native species of traditional use. In addition, the study also contributes to the genus Fridericia, since no previous studies on essential oil composition have been reported.
\end{abstract}

Keywords: Fridericia florida; Fridericia chica; CG-EM; monoterpenes; sesquiterpenes

\section{Composição química dos óleos essenciais de folhas de Fridericia florida DC. E Fridericia chica (Bonpl.).}

Resumo: Este projeto de pesquisa identificou a composição química dos óleos essenciais de folhas frescas de Fridericia florida DC L. G Lohman e Fridericia chica (Bonpl) (Bignoniaceae). Os óleos essenciais foram obtidos através de destilação por arraste de vapor. A identificação foi realizada por cromatografia gasosa com um espectrômetro de massas acoplado (CG-EM). Em seguida, os índices de retenção e os espectros de massas obtidos foram comparados com os valores relatados na literatura. Neste estudo, obteve-se um rendimento de $0,033 \%$ e foram identificados $96,78 \%$ (Fridericia florida) e 91,95\% (Fridericia chica) das respectivas composições totais relativas. Para Fridericia florida, 13 compostos foram identificados, sendo o limoneno o componente principal $(72,17 \%)$. Para Fridericia chica, 11 compostos foram identificados, sendo o germacreno D o componente principal (63,77\%). Esses resultados contribuem para a fitoquímica de ambas as espécies, ao identificar os componentes voláteis em espécies nativas de uso tradicional. Além disso, o estudo também contribuiu com novos dados sobre o gênero Fridericia, uma vez que não havia estudos prévios sobre a composição do óleo essencial dessas plantas.

Palavras-chave: Fridericia florida; Fridericia chica; CG-EM; monoterpenos; sesquiterpenos 


\section{Introducción}

El género Fridericia (sinonimia: Arrabidaea) (Lohman y Taylor, 2014) cuenta con aproximadamente 239 especies según The Plant List (2010), de las cuales 23 se encuentran en Colombia. Forma parte de la familia Bignoniaceae con cerca de 120 géneros y 800 especies distribuidas a nivel mundial, principalmente en las regiones tropicales; se encuentra con mayor concentración en los bosques húmedos de tierras bajas. Algunas de las especies del género se caracterizan por ser lianas con zarcillos terminales y flores en forma de trompeta de color blanco, rojo, rosa y púrpura (Forero, Betancur, Galeano y Aguirre, 2009).

Las investigaciones realizadas sobre este género han estado enfocadas en el efecto antimicrobiano (Moreira y Silva, 2016) y antifúngico (Alcerito et al., 2002) de la Fridericia platyphylla (sinonimia: Arrabidaea Brachypoda) (The Plant List, 2010), en la actividad antioxidante de la Fridericia samydoides (Pauletti Bolzani y Marx, 2003), la actividad contra el Trypanosoma cruzi de la Fridericia triplinervia (Leite, Oliveira, Lombardi, Filho y Chiari, 2006), y en diversas aplicaciones de la Fridericia chica (Carvalho, 2012; Miranda et al., 2017; Siraichi et al., 2013).

Fridericia chica y Fridericia florida son lianas usadas como colorantes tradicionales por comunidades indígenas y artesanas en diferentes regiones de Colombia (Devia, 2007; Klinger et al., 2000). Fridericia chica tiene un amplio uso etnobotánico, de manera que se emplea para curar heridas, fiebre, tratar enfermedades en los riñones, hígado, piel, inflamación (Costa et al., 2015) ulceras gástricas, anemia y herpes (Mafioleti, Ferrerirada, Colodel, Flach y Tabajara of Oliveira, 2013).

Debido a la variedad de usos con los que cuenta, se han realizado diversas investigaciones en las que se comprueban sus propiedades medicinales. Se encuentra que presenta actividad antifúngica (Ramos et al., 2008), anti Tripanosoma cruzi (Miranda et al., 2017) antiinflamatoria, antiangiogénica, antitumoral (Carvalho, 2012), antianémica, cicatrizante (Guedes et al., 2013) y fotoprotectora (Siraichi et al., 2013). Sus hojas se usan como colorante por los indígenas zenú en Córdoba en el teñido del sombrero vueltiao (Cabildo Mayor Regional del pueblo Zenú, 2014), y el producto obtenido por su fermentación lo usan como pintura corporal los indígenas del Orinoco y el Amazonas (Uscategui, 1961). Se ha identificado que los principales compuestos que contribuyen a la propiedad colorante de esta especie son de tipo 3- deoxiantocianos (Devia et al., 2002).

La Fridericia florida registra escasos estudios fitoquímicos, sus hojas presentan actividad citotóxica (Mesquita, Paula, Pessoa y de Moraes, 2009) y actividad antiinflamatoria y analgésica (Mourão et al., 2017); forma parte de las especies estudiadas como fuente promisoria de colorantes en el trapecio amazónico de Colombia (Klinger et al., 2000).

Los estudios realizados sobre aceites esenciales en la familia Bignoniaceae indican actividad antimicrobiana en la Mansoa difficilis con el 1-octen3-ol (49.65\%), dialil di- y trisulfuro (0.85 y $0.37 \%$, respectivamente) (Skelding et al., 2012), y en $\mathrm{Ja}$ caranda acutifolia, compuesta, principalmente, por ácidos grasos, con el ácido n-tetradecanoico (como compuesto mayoritario) (17.48\%) (Mostafa, Eldahshan y Nasser, 2014).

En este artículo se presenta la caracterización y comparación de la composición química del aceite esencial de hojas de Fridericia florida DC. L. G Lohman y Fridericia chica (Bonpl), especies nativas de uso tradicional, de manera que es el primer aporte al estudio de metabolitos volátiles de estas dos especies pertenecientes al género Fridericia.

\section{Materiales y métodos}

\section{Recolección e identificación de material vegetal}

Las hojas de Fridericia florida se recolectaron en el mes de octubre del 2016 en San Martín de Amacayacu (Amazonas). Una muestra testigo se envío al Herbario Forestal de la Universidad Distrital (UDBC), la cual fue identificada como Fridericia florida DC. L.G Lohman (Bignoniaceae). En cuanto a la Fridericia chica sus hojas fueron recolectadas en el mes de febrero del 2017 en Honda (Tolima), identificada previamente en el Herbario Nacional 
Colombiano como Arrabidaea chica H. B. K. (Verlot) (Bignoniaceae) $)^{1}$.

\section{Extracción de aceites esenciales}

Se realizó la extracción de los aceites esenciales por medio de destilación por arrastre con vapor de agua durante tres horas a partir de $300 \mathrm{~g}$ de hojas frescas de cada especie, obteniéndose $0,1 \mathrm{ml}$ de cada aceite, los cuales fueron solubilizados en $1 \mathrm{~mL}$ de cloroformo para su análisis por cromatografía de gases acoplada a espectrometría de masas (cg-em).

\section{Análisis de composición química en aceites esenciales}

El análisis de la composición química de los aceites esenciales se hizo por cg-em con un cromatógrafo shimadzu qp2010 plus empleando una columna capilar de sílice fundida, hp-5ms (J \& W Scientific, Folsom, CA, EE. UU.) de $60 \mathrm{~m} \mathrm{x} \mathrm{0,25} \mathrm{mm}$ con tamaño de partícula de $0.25 \mu \mathrm{m}$; como gas de arrastre se empleó helio grado 5.0 con un flujo de $1.2 \mathrm{~mL} / \mathrm{min}$. La programación de temperatura del horno fue de $40{ }^{\circ} \mathrm{C}(5 \mathrm{~min})$ con incrementos de 4 ${ }^{\circ} \mathrm{C} / \mathrm{min}$ hasta $160{ }^{\circ} \mathrm{C}, 2.5^{\circ} \mathrm{C} / \mathrm{min}$ hasta $220{ }^{\circ} \mathrm{C}$ y $8{ }^{\circ} \mathrm{C} / \mathrm{min}$ hasta $280^{\circ} \mathrm{C}(4 \mathrm{~min})$. Los espectros de masas se obtuvieron por ionización electrónica con una energía de $70 \mathrm{eV}$. Las temperaturas de la cámara de ionización y de la línea de transferencia fueron de 230 y $325^{\circ} \mathrm{C}$, respectivamente.

La identificación de la composición química de los aceites esenciales se llevó a cabo por medio de los espectros de masas reportados en la librería nist-08 y por comparación de los índices de retención (IR) experimentales y teóricos reportados en la literatura por R. Adams (2007). Para la determinación de los IR experimentales se tuvo en cuenta los tiempos de retención de una serie de hidrocarburos alifáticos en una solución estándar $\left(\mathrm{C}_{10}\right.$ a C20, Dr. Ehrenstorfer) analizada bajo las mismas condiciones de los aceites esenciales.

1 Sinonimia de Fridericia chica (Bonpl.) L. G. Lohmann (Lohman y Taylor, 2014).

\section{Resultados y discusión}

El método de destilación por arrastre con vapor permitió obtener, para cada una de las especies, $0,1 \mathrm{ml}$ de aceite esencial, con un rendimiento del $0.033 \%$. En la (Tabla 1) se presentan los compuestos identificados en Fridericia chica y Fridericia florida por CG-EM, la identificación de los índices de retención y la comparación de los espectros de masas conlalibreríanisT08.Sereportanloscompuestoscon porcentaje de coincidencia mayor al 80\%, así como se muestran los porcentajes de área que corresponden al porcentaje relativo de abundancia de cada componente dentro del aceite esencial.

En Fridericia chica se identificaron 11 compuestos (91.95\%) correspondientes al 8.77\% monoterpenoides y $83.18 \%$ sesquiterpenoides. Como componentes mayoritarios se destacan el monoterpeno limoneno, con porcentaje de área de $2.58 \%$, y el monoterpeno oxigenado R-carvona con $6.19 \%$ de área. Entre los sesquiterpenos se destacan germacreno -D con $63.77 \%$, bourboneno con $6.25 \%$ y $\beta$-elemeno con $5.00 \%$ de área. El sesquiterpeno oxigenado que se identificó es el germacreno D-4ol con $0.25 \%$ de área.

En Fridericia florida se identificaron 13 compuestos $(96.78 \%)$ correspondientes a $81.49 \%$ monoterpenoides y $15.29 \%$ sesquiterpenoides. Los monoterpenos identificados son el $\beta$-mirceno, limoneno y $\beta$-ocimeno, donde el limoneno es el componente mayoritario ( $72.17 \%$ de área). Entre los monoterpenos oxigenados se destaca p-timol, verbenona y R-carvona con el mayor porcentaje de área (7.18\%). Entre los sesquiterpenos se logró identificar el germancreno-D con una abundancia relativa del $11.18 \%$.

La comparación de los aceites esenciales obtenidos permite inferir que las dos especies tienen compuestos en común. Sin embargo, se presenta una significativa variación en el porcentaje de área relativo y la variabilidad de cada uno. La Fridericia florida posee una mayor cantidad de monoterpenos (6) que la Fridericia chica (2); en cuanto a sesquiterpenos la primera especie presenta una menor cantidad (7) que la segunda (9). Es posible evidenciar una diferencia entre los compuestos mayoritarios: el limoneno en la 
Fridericia florida tiene un porcentaje de área de $72.17 \%$, y en la Fridericia chica de $2.58 \%$. Por su parte, el germacreno D en la Fridericia chica tiene un porcentaje de área de $63.77 \%$, y en la Fridericia florida de $11.18 \%$.

Se han reportado estudios de composición de la familia Bignoniaceae (p. ej., el caso del género Mansoa con $M$. difficilis y $M$. alliaceae) que se caracterizan por tener sulfuros de alilo y dialilo, comúnmente determinados en especies del género Allium (Guilhon et al., 2012; Zoghbi, Oliveira y Guilhon, 2009). El 1-octen-3-ol es común en flores de Jacaranda accutifolia (Ueyama, Hashimoto y Furukawa, 1989) y de Tecoma grandiflora y en hojas y corteza de Tabebuia rosea (Oloyede Oladosu, Shodia y Oloyade, 2010).

Tabla 1. Composición química de los aceites esenciales de Fridericia chica Y Fridericia florida

\begin{tabular}{|c|c|c|c|c|}
\hline Compuesto $^{a}$ & $\mathbf{I R}^{\mathbf{b}}$ & $I^{C}$ & \%Composición Fridericia chica & \% Composición Fridericia florida \\
\hline$\beta$-Mirceno & 981 & 984 & 0.00 & 0.57 \\
\hline Limoneno & 1016 & 1031 & 2.58 & 72.17 \\
\hline$\beta$-Ocimeno & 1026 & 1040 & --- & 0.66 \\
\hline R-Carvona & 1245 & 1242 & 6.19 & 7.18 \\
\hline p-Timol & 1288 & 1290 & 0.00 & 0.19 \\
\hline Verbenona & 1195 & 1204 & 0.00 & 0.72 \\
\hline Copaeno & 1384 & 1376 & 0.28 & 0.00 \\
\hline Bourboneno & 1393 & 1374 & 6.25 & 1.23 \\
\hline$\beta$-Elemeno & 1398 & 1391 & 5.00 & 1.29 \\
\hline$\beta$-Farneseno & 1460 & 1458 & 2.27 & 0.24 \\
\hline Aromadendreno & 1472 & 1461 & 2.24 & 0.31 \\
\hline Germacreno D & 1492 & 1480 & 63.77 & 11.18 \\
\hline Germacreno B & 1507 & 1503 & 2.52 & 0.45 \\
\hline$\delta$-Cadineno & 1531 & 1524 & 0.60 & 0.00 \\
\hline Germacreno D-4-ol & 1586 & 1574 & 0.25 & 0.59 \\
\hline Total & & & 91.95 & 96.78 \\
\hline Monoterpenoides & & & 8.77 & 81.49 \\
\hline Sesquiterpenoides & & & 83.18 & 15.29 \\
\hline
\end{tabular}

a. Orden de elución en columna HP-5MS b Índice de retención calculado c Índice de retención de referencia (Adams, 2007).

El aceite esencial de las flores de Jacaranda accutifolia (Mostafa et al., 2014) es el único de los reportados que presenta similitudes con la composición de las hojas de Fridericia florida y Fridericia chica, y tienen en común germacreno $\mathrm{D}$, farneseno, elemeno y cadineno. En relación con las especies del género Fridericia se conoce muy poca información sobre la composición de los aceites esenciales.
Los compuestos mayoritarios en ambas especies presentan diferentes actividades biológicas según lo reportado en la literatura. El limoneno es antiinflamatorio, inmunomodulador, previene la obstrucción branquial y con actividad quimiopreventiva y quimioterapéutica contra diferentes tumores en modelos animales (De Cássia, Andrade y De Sousa, 2013). Presenta un efecto antimicrobiano 
frente a hongos (Cryptococcus neoformans) y bacterias (S. aureus, E. coli, Pseudomonas aeruginosa entre otras) (Van Vuuren et al., 2007).

Debido a su alto porcentaje en Fridericia flori$d a(72.17 \%)$, el limoneno puede dar a la planta un amplio rango de aplicaciones aún por investigar. El germacreno D tiene efectos contra el herbivorismo, actividad insecticida frente a los mosquitos y repelente para áfidos (Noge y Becerra, 2009). Adicionalmente, en combinación con otros terpenos como, por ejemplo, el cariofileno, presenta actividad antibacteriana (E. coli, Enterobacter spp o Bacillus spp, entre otras), y antifúngica (Fusarium spp o Aspergillus spp, entre otros) (Sahin et al., 2004). Al ser este el componente mayoritario en las hojas de Fridericia chica (63.77\%) podría contribuir a la actividad antimicrobiana previamente estudiada en los metabolitos fijos de la especie (Mafioleti et al., 2013; Moreira y Silva, 2016; Ramos et al., 2008).

Otros compuestos minoritarios que se encuentran en ambas especies, como, por ejemplo, la carvona que presenta actividad antiinflamatoria (Oloyede et al., 2010) y el $\beta$-elemeno para el que se ha determinado actividad antitumoral (Hua, Xianping, Linlin y Shu, 1997) pueden aportar a las propiedades biológicas de las dos especies vegetales.

\section{Conclusiones}

Se identificó por primera vez la composición química de metabolitos volátiles presentes en dos especies del género Fridericia. El análisis por CG-EM del aceite esencial en la Fridericia florida mostró que hay mayor proporción de monoterpenos, con el limoneno como compuesto mayoritario (72.17\%), mientras que en la Fridericia chica hay mayor proporción de sesquiterpenos, con el germacreno D como compuesto mayoritario (63.77\%). La mayoría de los compuestos determinados son comunes para ambas especies, presentándose variaciones en cuanto a las proporciones en que se encuentran.

\section{Referencias}

[1] Adams, R. (2007). Identification of Essential Oil Components by Gas Chromatography/Mass Spectroscopy. EE. UU.: Allured Publishing Corporation.
[2] Alcerito, T., Barbo, F. E., Negri, G., Santos, D., Meda, C. I., Young, M. C. y Blatt, C. T. (2002). Foliar Epicuticular Wax of Arrabidaea Brachypoda: Flavonoids and Antifungal Activity. Biochemical Systematics and Eco$\log y, 30(7), 677-683$.

[3] Cabildo Mayor Regional del Pueblo Zenú. (2014). Diagnóstico comunitario y líneas de acción para el plan de salvaguarda étnica del pueblo Zenú capitulo Córdoba-Sucre y dispersos (Tomo I). (Convenio Interadministrativo N.o M-923 de 2013 celebrado Entre La Nación y Ministerio del interior: 110-119). Córdoba, Colombia: Cabildo Mayor Regional del Pueblo Zenú.

[4] Carvalho, A. F. (2012). Avaliação das atividades antiinflamatória, antiangiogênica e antitumoral de extratos da Arrabidaea chica (Humb. \& Bonpl.) B. Verlot (tesis doctoral). Universidad Federal de Minas Gerais, Bello Horizonte, Brasil.

[5] Costa, I. G., Leonti, M., Thor, J., Ferrier, J., Rapinski, M., Pavoa, I. M. ... y Tabajara de Oliverira, D. (2015). Ethnobotanical Study of Medicinal Plants by Population of Valley of Juruena Region, Legal Amazon. Journal of Ethnopharmacology, 173(40), 32-34.

[6] De Cássia, R., Andrade, L. y De Sousa, D. (2013). A Review on Anti-Inflammatory Activity of Monoterpenes. Molecules, 18(1), 1227-1254.

[7] Devia, B. (2007). Determination de L'emploi d'une nouvelle source de colorants rouges a L'epoque précolobienne: Arrabidaea chica H.B.K. Bulletin, 31(05), 297-321.

[8] Devia, B., Tits, M., Llabres, G., Wouters, J., Dupont L, Escribano, M., ... y Angenot, L. (2002). New 3-Deoxyanthocyanidins from Arrabidaea Chica Leaves. Phytochemical Analysis, 114-120.

[9] Forero, E., Betancur, J., Galeano, G. y Aguirre, J. (2009). Gentry, A. H. Bignoniaceae. Flora de Colombia, 25(Monografía), 72-136.

[10] Guedes, F. A., Carvalho, C., Costa, J. C., Ferreira, J. y Silva F. (2013). Comprovação do potencial medicinal de Arrabidaea chica (Bignoniaceae). Scientia Prim, 1(1), 15-20.

[11] Guilhon, G., Sarmento, E., Silva, L., Graças, M., Santos, I. y Trovatti, A. (2012). Volatile and Non-Volatile Compounds and Antimicrobial Activity Of Mansoa Difficilis (Cham.) Bureau \& K. Schum. (Bignoniaceae). Quimica Nova, 35(11), 2249-2253.

[12] Hua, Y., Xianping, W., Linlin, Y. y Shu, Z. (1997). The Antitumor Activity of Elemene is Associated With Apoptosis. Journal of Cancer Research, 18(3), 83-88.

[13] Klinger, W., Pinzón, C., Pachón, M., Rojas, F. y Aragón, J. (2000). Estudio de las especies promisorias 
productoras de colorante en el trapecio amazónico. Colombia Forestal, 11(5), 15-33.

[14] Leite, J. P., Oliveira, A. B., Lombardi, J. A., Filho, J. D. y Chiari E. 2006. Trypanocidal activity of triterpenes from Arrabidaea triplinervia and derivatives. Biological \& Pharmaceutical Bulletin, 29(11), 2307-2309.

[15] Lohman, L. G. y Taylor, C. M. (2014). A New Generic Classification of Tribe Bignonieae (Bignoniaceae). Annals of the Missouri Botanical Garden, 99, 348-489.

[16] Mafioleti, L., Ferrerirada, I., Colodel, E., Flach, A. y Tabajara of Oliveira, D. (2013). Evaluation of the Toxicity and Antimicrobial Activity of Hydroethanolic Extract of Arrabidaea chica (Humb. \& Bonpl.) B. Verl. Journal of ethnopharmacology, 150(2), 576-582.

[17] Mesquita, M., de Paula, E., Pessoa, C. y de Moraes, M. (2009). Cytotoxic Activity of Brazilian Cerrado Plants Used in Traditional Medicine Against Cancer Cell Lines. Journal of ethnopharmacology, 123(3), 439-445.

[18] Miranda, N., Passarella, A., Novello, C., Nakamura, T. U., of Oliveira, S., Prado, B. ... y Nakamura, C. V. (2017). Pheophorbide a, a Compound Isolated from the Leaves of Arrabidaea Chica, Induces Photodynamic Inactivation of Trypanosoma Cruzi. Photodiagnosis and Photodynamic Therapy, 16,: 256-265.

[19] Moreira, L. y Silva, S. (2016). Ação inibitória do Crajiru Arrabidaea chica (Humb. \& Bonpl.) B. Verlot sobre Staphylococcus sp, como microorganismo oportunista no tratamiento da acne vulgar. Revista de Publicações da FAAr, 2(2), 210-232.

[20] Mostafa, N., Eldahshan, O. y Nasser, A. (2014). Chemical Composition and Antimicrobial Activity of Flower Essential Oil of Jacaranda acutifolia Juss. Against, Food-Borne Pathogens, European Journal of Medicinal Plants, 6(2), 62-69.

[21] Mourão, P., Macedo, J., Da Silva, A., Da silva, C., Marques, D. y Da Cunha, M. (2017). Pharmacological Analysis of Two Ethanolic Extracts from Species of Fridericia Genus. Revista da Universidade Vale do Rio Verde, 15(2), 122-131.

[22] Noge, K. y Becerra, J. (2009). Germacrene D, A Common Sesquiterpene in the Genus Bursera (Burseraceae). Molecules, 14(1), 5289-5294.

[23] Oloyede, G. K., Oladosu, I. A., Shodia, A. F., Oloyade, O. O. (2010). Cytotoxic Effects of Tabebuia rosea Oils
(Leaf and Stem Bark). Archives of Applied Science Research, 2(3), 127-130.

[24] Pauletti, P. M., Bolzani, V. S. y Marx, M. C. (2003). Constituintes químicos de Arrabidaea samydoides (Bignoniaceae). Journal Química Nova, 26(5), 641643.

[25] Ramos, W. L., Do Nascimento, L., Quignard, E., Dos Santos, J. M., Carrera J. O. y Albuquerque, S. (2008). Arrabidaea chica (HBK) Verlot: Phytochemical Approach, Antifungal and Trypanocidal Activities. Revista Brasileira de Farmacognosia, 18(4), 544-548.

[26] Sahin, F., Gulluce, M., Daferera, D., Sokmen, A., Sokmen, M., Polissiou, M. ... y Ozer, H. (2004). Biological Activities of the Essential Oils and Methanol Extract of Origanum vulgare ssp. Vulgare in the Eastern Anatolia Region of Turkey. Food Control, 15(7), 549-557.

[27] Siraichi, J. T., Pedrochi, F., Natali, M. R., Ueda-Nakamura, T., Filho, B. P., Bento, A. C. ...Nakamura, C. V. (2013). Ultraviolet (UVB and UVA) photoprotector activity and percutaneous penetration of extracts obtained from Arrabidaea chica Revista Brasileira de Ciências Farmacêuticas, 67(10), 1179-1184.

[28] Skelding, G. M., Sarmento, E., da Silva, L., das Graças, M., Santos, I. y Trovatti, A. P. (2012). Volatile and Non-Volatile Compounds and Antimicrobial Activity of Mansoa difficilis (Cham.) Bureau \& K. Schum. (Bignoniaceae). Química Nova, 35(10), 2249-2253.

[29] The Plant List. (2010). Arrabidaea. Recuperado de http://www.theplantlist.org/tpl/search?q=Arrabidaea.

[30] Ueyama, Y., Hashimoto, S. y Furukawa, K. (1989). The Essential Oil from the Flowers of Campsis grandiflora (Thumb.) K. Schum. from China, China, Flavour and Fragrance. Journal, 4, 103-107.

[31] Uscátegui, N. (1961). Algunos colorantes vegetales usados por las tribus indígenas de Colombia. Revista Colombiana de Antropología, 10(1), 333-340.

[32] Van Vuuren, S. F. y Viljoen, A. (2007). Antimicrobial activity of limonene enantiomers and 1,8-cineole alone and in combination. Flavour and Fragrance Journal, 22(6), 540-544.

[33] Zoghbi, M., Oliveira, J., Guilhon, G. (2009). The Genus Mansoa (Bignoniaceae): A Source of Organosulfur Compounds. Revista Brasileña de Farmaconogsia, 19(3), 795-804. 




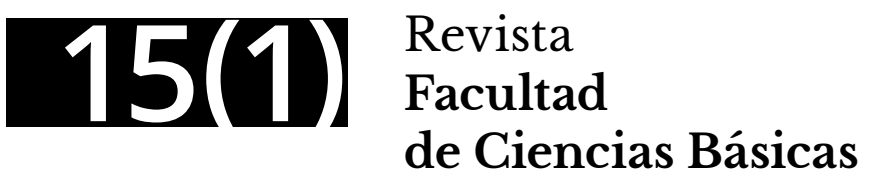

| Volumen 15(1) |' Enero-junio 2019 | ISSN: 1900-4699 |' ISSN-e: 2500-5316 | 